\title{
Scales of String Theory 团
}

\author{
C P Bachas \\ Laboratoire de Physique Théorique de l' Ecole Normale Supérieure, \\ 24 rue Lhomond, F-75231 Paris Cedex 05, France \\ bachas@physique.ens.fr
}

\begin{abstract}
I review the arguments in favor of/against the traditional hypothesis that the Planck, string and compactification scales are all within a couple of orders of magnitude from each other. I explain how the extreme brane-world scenario, with $\mathrm{TeV}$ type I scale and two large (near millimetric) transverse dimensions, creates conditions analogous to those of the energy desert and is thus naturally singled out. I comment on the puzzle of gauge coupling unification in this context.
\end{abstract}

\section{Introduction}

String/M theory [1, 2] has a single dimensionful parameter and a large number of dynamical moduli. The expectation values of these moduli determine the semiclassical properties of the vacuum, and in particular the masses of its Kaluza-Klein and Regge excitations. It is hoped that some still unknown dynamical mechanism ultimately selects the (unique?) vacuum in which we live, thereby fixing also the scales of the new physics. A more down-to-earth approach is to rely on arguments of theoretical plausibility, on direct experimental limits and on indirect experimental hints. In the wake of the 'duality revolution' there has been a revival of interest on the question of scales [3-17] which I will try to review in the present talk. 目

The conventional (and conservative) hypothesis is that the string, compactification and Planck scales lie all to within two or three orders of magnitude from each other, and are hence far beyond direct experimental reach. The non-gravitational physics at lower energies is believed to be described by a renormalizable supersymmetric quantum field theory (SQFT), which must include the Minimal Supersymmetric Standard Model (MSSM). Faith in this hypothesis has been bolstered by the following wellknown facts : (i) Softly broken SQFTs can indeed be extrapolated consistently to nearPlanckian energies without destabilizing the electroweak scale ; (ii) the minimal (or 'desert') unification assumption is in remarkable agreement with some of the measured low-energy parameters of our world, and (iii) the hypothesis is almost automatic within the weakly-coupled heterotic string.

The negative side of the coin is that this story is at best incomplete : coupling the broken SQFT to (super)gravity gives rise to vacuum instabilities, including an unacceptably-large cosmological term. As has been (re)appreciated in recent years, our knowledge of the gravitational interaction is in fact also very limited in another

$\ddagger$ Talk given at the Strings 99 Conference, Potsdam, July 1999. To be published in Class. Quantum Grav. 17 (2000) 1.

$\S$ The audio version and transparencies can be found in [2]. 
way: Einstein's classical theory has not been tested experimentally at distances shorter than the macroscopic (millimeter or $10^{-3} \mathrm{eV}$ ) regime [18]. Since this is also the observational upper bound on the cosmological constant [19], it is very tempting to speculate that a resolution of the associated long-standing puzzle will require a drastic modification of gravity at such scales. Recent proposals of a higher-dimensional gravity [6. 14 do not seem to point to a resolution of the problem [20], while more drastic modifications like millimeter-sized fundamental strings [21] are hard to accomodate in a consistent theoretical framework. The problem of gravitational (in)stability is, in any case, an important motivation for pursuing alternatives to the conventional heterotic compactification scheme.

Two other important motivations are (i) that compactification scenaria which confine gauge interactions on a brane arise, as we have learned, very naturally in controllable corners of the moduli space of M-theory, and (ii) that they can bring string and Kaluza-Klein physics closer to experiment. Furthermore, the extreme brane-world scenario with TeV type-I string scale [4, [7] and a (near-millimetric ?) transverse space of dimension two, is singled out as I will explain in this talk. The logarithmic sensitivity on the transverse size [10, 11] sets a stage similar to that of the conventional energy desert : it makes hierarchies of scales very natural, and allows reliable (model-independent) calculations of the effective parameters in the brane theory. The apparent unification of the measured low-energy gauge couplings, on the other hand, has not found a convincing explanation outside the traditional energy-desert scenario yet. Since this is arguably [22] the only clear quantitative hint for physics beyond the Standard Model, I will start and end my discussion from this point.

\section{Hints of Supersymmetric Unification}

The well-known observation [23, 24, 25] is that if one extrapolates the three gauge couplings of $S U(3) \times S U(2) \times U(1)$ using the $\beta$-funcions of the MSSM they meet at a scale $M_{U} \simeq 2 \times 10^{16} \mathrm{GeV}$. This is consistent with the one-loop formulae

$$
\alpha_{i}{ }^{-1}(\mu)=\alpha_{U}{ }^{-1}+b_{i} \log \frac{\mu}{M_{U}}+\Delta_{i}
$$

where $b_{i}$ are the $\beta$-function coefficients, and $\alpha_{U}$ is the fine structure constant at $M_{U}$. The threshold corrections $\Delta_{i}$ parametrize our ignorance of the details of the theory at the unification scale, and of the details of supersymmetry breaking. Assuming that the $\Delta_{i}$ are negligible, and treating $M_{U}$ and $\alpha_{U}$ as input parameters, we have one prediction which is verified by LEP data at the level of a few percent.

In the minimal heterotic unification there is furthermore one extra relation [26] between the input parameters and the experimental value of $M_{\text {Planck. }}$ It implies $M_{U} \simeq 5 \times 10^{17} \mathrm{GeV}$, which on the (appropriate) logarithmic scale is a second successful prediction of the theory at the level again of a few percent [27, 28]. Put differently: there was no a priori reason why the extrapolated low-energy couplings should not have met, if at all, at say $10^{35} \mathrm{GeV}$ ! It is important here to realize that it is the very existence of the perturbative desert which renders the above predictions meaningful and robust. Threshold effects make a few-percent correction to differences of couplings only because the logarithm in equations (1) is very large. It would be impossible to ignore the nitty-gritty details of the model if the unification scale were say instead at $100 \mathrm{TeV}$. 
Similar arguments can be given for the mass matrices of quarks and leptons. Minimal assumptions (such as discrete symmetries and Higgs-field content) determine boundary conditions for Yukawa couplings, which can then be evolved with the equations of the renormalization group. The agreement with low-energy data is suggestive 25, though less compelling than for the gauge-coupling constants. Finally, I should mention that the existence of the superheavy scale $M_{U}$ is indirectly supported by two other independent pieces of data: the very long lifetime of the proton [29] and the extraordinary smallness of neutrino masses [30].

\section{Weakly-coupled Heterotic String}

Let me proceed next to the theoretical arguments that make the SQFT hypothesis quasi-automatic within the weakly-coupled heterotic string. Both the graviton and the perturbative gauge bosons live in this case in the ten-dimensional bulk, and interact through the sphere diagram at tree-level. The four-dimensional Yang-Mills and Einstein actions therefore read

$$
\mathcal{L}_{\text {gauge }} \sim \frac{\left(r M_{h}\right)^{6}}{g_{h}^{2}} \operatorname{tr} F^{2} \text { and } \quad \mathcal{L}_{\text {grav }} \sim \frac{r^{6} M_{h}^{8}}{g_{h}^{2}} \mathcal{R},
$$

where $M_{h}$ and $g_{h}$ are the heterotic string scale and string coupling constant, while $r$ is the typical compactification radius. By virtue of T-duality $r$ can be always taken greater than, or equal to, the string length. From the coefficients of these actions we can read the four-dimensional gauge coupling and Planck mass with the result

$$
\alpha_{U} \sim \frac{g_{h}^{2}}{\left(r M_{h}\right)^{6}}
$$

and

$$
M_{h}^{2} \sim \alpha_{U} M_{\text {Planck }}^{2}
$$

Factors of 2's and $\pi$ 's in these relations are irrelevant for our arguments and have been dropped. I have also dropped the level of the corresponding Kac-Moody algebra which for all practical purposes is an integer of order one.

Assume now that (i) the bare gauge coupling is of order one, and (ii) the heterotic theory stays weakly coupled $\left(g_{h} \leq 1\right)$. Then the universal relation (1), which we have already encountered in the previous section, implies that the string scale is tied automatically to the Planck mass. Relation (3) on the other hand also implies that $r M_{h}$ cannot be much larger than one. Since it cannot be smaller than one by Tduality, it is necessarily of order one. Thus there is little leeway for abandoning the conventional scenario within the context of the weakly-coupled heterotic string.

We can of course try to relax one of the above two assumptions : either (i) allow $g_{h}$ to be hierarchically large and hope that the gauge sector still stays under control in some special models [31], or (ii) let $\alpha_{U}$ be hierarchically small and hope that the Standard Model gauge couplings will be driven to their measured values by the large threshold corrections of a higher dimensional field theory 32. 32

Such exotic possibilities have been motivated in the past by the search for classical vacua with broken low-energy supersymmetry. Known 'mechanisms' of continuous supersymmetry breaking [34, 35] indeed tie the breaking scale to the size of some

$\dagger$ Higher-dimensional thresholds have been discussed also in other contexts, see for example [33]. 
internal dimensions. Classical supersymmetry restoration can be furthermore argued to be singular, in string theory, on general grounds [36. It was thus suggested early on [37] that one (or more) radii of inverse size at the $\mathrm{TeV}$ would be required if the 'observed' supersymmetry breaking in nature were classical. Tree-level breaking, on the other hand, is at best an assumption of convenience - there is no reason in principle why the breaking in nature should not have a non-perturbative origin. Furthermore the classical mechanisms have not so far lead to new insights on the crucial problems of vacuum selection and stability. Thus, there seemed to be little theoretical motivation for abandoning the conventional compactification scheme, and its successful unification predictions, in heterotic string theory.

\section{Brane World and Type-I Theory}

One of the important developments of the 'duality revolution' has been the realization that various branes - Dirichlet branes [38, 39, or their dual heterotic fivebranes [40] and Horava-Witten walls [41] - can trap non-abelian gauge interactions in their worldvolumes. This has placed on a firmer basis an old idea [42 according to which we might be living on a brane embedded in a higher-dimensional world. The idea arises naturally in compactifications of type I theory [43], which typically involve collections of orientifold planes and D-branes. I will from now on restrict my discussion to this context, because in it the 'brane-world' scenario admits a fully perturbative string description. All other interesting possibilities (see for example [14, 15, 16]) involve some type of non-perturbative dynamics and are a priori harder to control.

In type I string theory the graviton (a closed-string state) lives in the tendimensional bulk, while open-string vector bosons are in general localized on lowerdimensional D-branes. Furthermore while closed strings interact to leading order via the sphere diagram, open strings interact via the disk diagram which is of higher order in the genus expansion. The four-dimensional Planck mass and Yang-Mills couplings therefore take the form

$$
\alpha_{U} \sim \frac{g_{\mathrm{I}}}{\left(\tilde{r} M_{\mathrm{I}}\right)^{6-n}}, \quad M_{\text {Planck }}^{2} \sim \frac{r^{n} \tilde{r}^{6-n} M_{\mathrm{I}}^{8}}{g_{\mathrm{I}}^{2}},
$$

where $r$ is the typical radius of the $n$ compact dimensions transverse to the brane, $\tilde{r}$ the typical radius of the remaining (6-n) compact longitudinal dimensions, $M_{\mathrm{I}}$ the type-I string scale and $g_{\mathrm{I}}$ the string coupling constant. By appropriate T-dualities we can again ensure that both $r$ and $\tilde{r}$ are greater than or equal to the fundamental string scale. T-dualities change $n$ and may take us either to Ia or to Ib theory (also called I or I', respectively) but I will not make a distinction between these two.

It follows from these formulae that (i) there is no universal relation between $M_{\text {Planck }}, \alpha_{U}$ and $M_{\mathrm{I}}$ anymore, and (ii) tree-level gauge couplings corresponding to different sets of D-branes have radius-dependent ratios and need not unify at all. Thus type-I string theory is much more flexible (and less predictive) than its heterotic counterpart. The fundamental string scale, $M_{\mathrm{I}}$, in particular is a free parameter, even if one insists that $\alpha_{U}$ be kept fixed and of order one, and that the string theory be weakly coupled. This added flexibility can be used to 'remove' the order-of magnitude discrepancy between the apparent unification and string scales of the heterotic theory [3], to lower $M_{\mathrm{I}}$ to an intemediate scale [12, 13 or even all the way down to its experimentally-allowed limit of order the $\mathrm{TeV}$, 1,7 . Keeping for instance $g_{\mathrm{I}}, \alpha_{U}$ and 
$\left(\tilde{r} M_{\mathrm{I}}\right)$ fixed and of order one, leads to the condition

$$
r^{n} \sim M_{\text {Planck }}^{2} / M_{\mathrm{I}}^{2+n}
$$

A $\mathrm{TeV}$ string scale would then require from $n=2$ millimetric to $n=6$ fermi-size dimensions transverse to our brane world. The relative weakness of gravity is in this picture attributed to the transverse spreading of gravitational flux.

\section{Experimental Bounds}

What has brought the brane-world idea into focus [6] was the realization that it cannot be a priori ruled out by the existing data, even in the most extreme case of ' $\mathrm{TeV}$-ish' string scale and millimmeter-size transverse dimensions. Gravity is hard to test at submillimeter distances because of the large background of residual electromagnetic interactions. The ratio for instance of the Van der Waals to Newtonian force between two hydrogen atoms a distance $d$ apart is [6]

$$
\frac{F_{\mathrm{VdW}}}{F_{\text {grav }}} \sim\left(\frac{1 m m}{d}\right)^{5} \text {. }
$$

At $d=10 \mu m$ Newton's force is thus ten orders of magnitude weaker than Van der Waals! As a result the present-day data 18 allows practically any modification of Newton's law, as long as it is of comparable strength at and screened beyond the millimeter range. This has been appreciated in the past in the context of gravitational axions 44], and similar bounds hold for light string moduli [45] or extra Kaluza-Klein dimensions [5, 6, 46].

Besides mesoscopic gravity experiments, there are two other types of direct experimental limits one should worry about : those coming from precision observables of the Standard Model, and those coming from various exotic processes. Precision tests of the SM and compositeness bounds cannot rule out in a model-independent way any new physics above the TeV-ish scale. Bounds for instance from LEP data on four-fermion operators, or bounds on dimension-five operators contributing to the $g-2$ of the electron/muon are safe, as long as the characteristic scale of the new physics is a few $\mathrm{TeV}$ [6]. Proton decay and other exotic processes could of course rule out large classes of low-scale models. There exist however plausible suppression mechanisms, such as bulk U(1) gauge symmetries which are spontaneously-broken at some distant brane [8, 47] and look like approximate global symmetries in our brane world. One type of model-independent exotic process is graviton emission in the bulk, which could be seen as missing-energy events in collider experiments [48]. The process is however suppressed by the four-dimensional Newton constant at low energies, and only becomes appreciable (as one should expect) near string scale where quantum gravity effects are strong.

None of these (or other) phenomenological considerations seems a priori fatal to the brane-world scenario, even in its extreme realization. Put together they will, however, probably make realistic type-I model building a very strenuous exercise indeed. 


\section{The Trasverse Desert}

Although $M_{\mathrm{I}}$ could lie anywhere between the Planck mass and the TeV, lowering it to the latter scale has two advantages : (i) it brings string physics within the reach of future acceleretor experiments, and (ii) it is a natural starting point for discussing the problem of the gauge hierarchy, which becomes now a question in the infrared [6, 11]. In a certain sense this extreme choice is antipodal to the energy-desert scenario: although the MSSM is a stable renormalizable field theory, we are shrinking its range of validity to one order of magnitude at most! Nevertheless, as I will now argue, these two scenaria share many common features when the number of large dimensions transverse to our brane is exactly two [10, 11].

The key feature of the SQFT hypothesis is that low-energy parameters receive large logarithmic corrections, which are effectively resummed by the equations of the Renormalization Group. The logarithmic sensitivity of parameters also generates naturally hierarchies of scales, and has been the key ingredient in all efforts to understand the origin of the gauge hierarchy in the past 44. Consider now the brane world scenario. The parameters of the effective brane lagrangian are dynamical open- and closed-string moduli with constant expectation values along the four noncompact space-time dimensions of our world. The closed-string moduli, $m_{a}$, are bulk fields whose expectation values will generically vary as a function of the transverse coordinates $\xi$. They include the dilaton, twisted-sector massless scalars, the metric of the transverse space etc. For weak type-I string coupling these variations can be described by a lagrangian of the form

$$
\mathcal{L}_{\text {bulk }}+\mathcal{L}_{\text {source }} \sim \int d^{n} \xi\left[\frac{1}{g_{\mathrm{I}}^{2}}\left(\partial_{\xi} m_{a}\right)^{2}+\frac{1}{g_{\mathrm{I}}} \sum_{s} f_{s}\left(m_{a}\right) \delta\left(\xi-\xi_{s}\right)\right] .
$$

Here $\mathcal{L}_{\text {bulk }}$ is a reduced supergravity Lagrangian while the sources are the D-branes and orientifolds which are localized at positions $\xi_{s}$ in the transverse space. The couplings $f_{s}\left(m_{a}\right)$ may vary from source to source - they can for instance depend on open-string moduli - and are subject to global consistency conditions. What is important is that they are weak in the type-I limit, leading to weak field variations,

$$
m_{a}(\xi)=m_{a}^{0}+g_{\mathrm{I}} m_{a}^{1}(\xi)+\cdots,
$$

with $m_{a}^{0}$ the (constant) average value, $m_{a}^{1}(\xi)$ given by a sum of Green's functions, and so on. For $n=2$ transverse dimensions the leading variation grows logarithmically with the size, $r$, of the transverse space. Since our Standard Model parameters will be a function of the moduli evaluated at the position of our brane world, they will have logarithmic sensitivity on $M_{\text {Planck }}$, very much like the (relevant) parameters of a supersymmetric renormalizable QFT. Similar sensitivity may occur even if $n>2$, as long as some of the 'bulk' moduli propagate in only two extra large dimensions.

The bulk supergravity Lagrangian receives both stringy and higher-genus corrections, but these involve higher derivatives of fields, and should therefore be negligible for moduli varying logarithmically over distances much larger than the string scale. The source functions, $f_{s}\left(m_{a}\right)$, will also be generically modified by such corrections - the D-branes have indeed string-scale thickness when probed by the supergravity fields [50]. Such source modifications can, however, be absorbed into boundary conditions for the supergravity equations, at the special marked points $\xi_{s}$. The situation thus looks (at least superficially) analogous to that prevailing under the SQFT hypothesis : large corrections to effective low-energy couplings can be 
in both cases resummed by differential equations subject to appropriate boundary conditions. Furthermore 'threshold corrections' parametrizing our ignorance of the detailed physics at distant branes - the analog of physics near the unification scale - have a small effect on the relative evolution of parameters, provided the transverse two-dimensional space is sufficiently large. Clearly $n=2$ is critical: for exactly one transverse dimension bulk fields vary linearly in space and one expects to hit strongcoupling singularities before $r$ can grow very large, while for $n>2$ the dynamics on our brane completely decouples from the dynamics elsewhere in the bulk.

\section{The Puzzle of Unification}

The logarithmic sensitivity of brane parameters with $r$ seems a natural setting for generating scale hierarchies dynamically, as in the case of renormalizable SQFT. Gauge dynamics on a given brane, for example, could become strong as the transverse space expands to an exponentially large size, thereby inducing gaugino condensation and supersymmetry breaking. The problems of vacuum selection and stability are, to be sure, still with us - the situation is, in this respect, neither better nor worse than in the traditional compactification scenario.

The apparent unification of the (MS)SM gauge couplings, on the other hand, has not yet found a convincing 'explanation' in this (or any alternative) context. The basic ingredients are, nevertheless, present [10] (see also [51, 52] for related ideas): (i) the logarithmic variation of bulk fields in the large transverse space can give equallyrobust predictions for brane parameters, as renormalization group running over the energy desert, (ii) some bulk fields (like twisted scalar moduli) have non-universal couplings to gauge fields that live on the same set of D-branes [53] - they can thus split their gauge couplings apart without separating them in transverse space, and (iii) the boundary condition imposing unification at high energy could be replaced by a boundary condition that these non-universally coupled bulk moduli vanish on some far-distant brane (for instance because of a non-perturbative superpotential). The main difficulty of these ideas is to explain why the coefficients of the logarithmic real-space evolutions should have the same (ratio of?) differences as the MSSM beta

functions. This is clearly required if we are to 'understand' the actual values of the SM gauge couplings, as measured at LEP energies.

\section{Aknowledgements}

I thank the organizers for the invitation to speak, and for organizing a marvellous strings 99 conference. This work was partially supported by the TMR contract ERBFMRX-CT96-0090. 


\section{References}

[1] M.B. Green, J.H. Schwarz and E. Witten, Superstring Theory (Cambridge U. Press, 1987); J. Polchinski, String Theory (Cambridge U. Press, 1998) ;

D. Olive and P. West eds., Duality and Supersymmetric Theories (Cambridge U. Press, 1998);

[2] http ://www.asi-potsdam.mpg.de/cgi-bin/viewit.cgi.

[3] E. Witten, Nucl. Phys. B471 (1996) 135, hep-th/9602070.

[4] J.D. Lykken, Phys. Rev. D54 (1996) 3693, hep-th/9603133.

[5] E. Carceres, V.S. Kaplunovsky and I.M. Mandelberg, Nucl.Phys. B493 (1997) 73, hepth/9606036.

[6] N. Arkani-Hamed, S. Dimopoulos and G. Dvali, Phy.s. Lett. B429 (1998) 263, hep-ph/9803315 and Phys. Rev. D59 (1999)086004, hep-ph/9807344.

[7] I. Antoniadis, N. Arkani-Hamed, S. Dimopoulos and G. Dvali, Phys. Lett. B436 (1998) 257, hep-ph/9804398

[8] G. Shiu and S.-H. H. Tye, Phys. Rev. D58 (1998) 106007, hep-th/9805157

[9] Z. Kakushadze and S.-H. Henry Tye, Nucl.Phys._B548 (1999) 180, hep-th/9809147.

[10] C. Bachas, JHEP 9811 (1998) 023, hep-ph/9807415; and in Fundamental Interactions : From Symmetries to Black Holes, J.-M. Frère et al eds., Brussels 1999, hep-th/9907023.

[11] I. Antoniadis and C. Bachas, Phys. Lett. B450 (1999) 83, hep-th/9812093

[12] K. Benakli, Phys.Rev. D60 (1999) 104002, hep-ph/9809582.

[13] C. Burgess, L.E. Ibanez and F. Quevedo, Phys.Lett. B447 (1999) 257, hep-ph/9810535

[14] L. Randall and R. Sundrum, Phus.Rev.Lett. 83 (1999) 3370, hep-ph/9905221 ; and Phys.Rev.Lett. 83 (1999) 4690, hep-th/9906064.

[15] I. Antoniadis and B. Pioline. Nucl. Phys. B550 (1999) 41, hep-th/9902055.

[16] K. Benakli and Y. Oz, hep-th/9910090.

[17] See also the talks by L. Ibanez, and by I. Antoniadis and A. Sagnotti.in this volume.

[18] J.C. Long, H.W. Chan and J.C. Price, Nucl.Phys. B539 (1999) 23, hep-ph/9805217.

[19] S. Weinberg, Rev. Mod. Phys. 61 (1989) 1.

[20] See however E. Verlinde, in this volume; C.P. Burgess, R.C. Myers and F. Quevedo, hep-th/9911164

[21] R. Sundrum, JHEP 9907 (1999) 001, hep-ph/9708329; T. Tomaras, private communication.

[22] See for instance S. Dimopoulos, in History of Original Ideas and Basic Discoveries in Particle Physics ( Erice 1994), hep-ph/9412297.

[23] H. Georgi, H. Quinn and S. Weinberg, Phys. Rev. Lett. 33 (1974) 451.

[24] S. Dimopoulos, S. Raby and F. Wilczek, Phys. Rev. D24 (1981) 1681; S. Dimopoulos and H. Georgi, Nucl. Phys. B193 (1981) 150; L. Ibanez and G.G. Ross, Phys. Lett. B106 (1981) 439; N. Sakai, Z. Phys. C11 (1981) 153.

[25] More recent discussions are given in P. Langacker and N_Ponsky,Phys.Rev. D47 (1993) 4028, hep-ph/9210235; and Phys.Rev. D49 (1994) 1454, hep-ph/9306205;

M. Carena, S. Pokorski and C.E.M. Wagner, Nucl. Phys. B406 (1993) 59, hep-ph/9303202 ; for fermion masses see also M. Carena, S. Dimopoulos, S. Raby and C.E.M. Wagner, Phys. Rev. D52 (1995) 4133, and references therein.

[26] P. Ginsparg, Phys. Lett. B197 (1987)139.

[27] V. Kaplunovsky, Nucl. Phys. B307 (1988) 145; L. Dixon, V. Kaplunovsky and J. Louis, Nucl. Phys. B329 (1990) 27.

[28] For a review see K. Dienes, Phys. Rep. 287 (1997) 447, and references therein.

[29] For a review see G. Costa and F. Zwirner, Riv. Nuovo Cim. 9 (1986) 1.

[30] For a review see G. Altarelli and F. Feruglio, hep-ph/9905536, to appear in Physics Reports.

[31] I. Antoniadis, Phys. Lett. B246 (1990) 377.

[32] C. Bachas, unpublished (1995).

[33] T.R. Taylor and G. Veneziano, Phys. Lett. B212 (1988) 147 ; K. R. Dienes, E. Dudas and T. Gherghetta. Phys. Lett. B436 (1998) 55, hep-ph/9803466; Z. Kakushadze and T.R. Taylor, hep-th/9905137.

[34] J. Scherk and J.H. Schwarz, Phys. Lett. B82 (1979) 60; and Nucl. Phys. B153 (1979) 61 ; R. Rohm, Nucl. Phys. B237 (1984) 553 ;

S. Ferrara, C. Kounnas and M. Porrati, Nucl. Phys. B304 (1988) 500 ;

S. Ferrara. C. Kounnas, M. Porrati and F. Zwirner, Nucl.Phys. B318 (1989) 75.

[35] C. Bachas. hep-th/9503030; and in Topics in QFT, D. Tchrakian ed. (World Scientific, 1995), hep-th/9509067; 
M. Berkooz, M.R. Douglas and R.G. Leigh, Nucl.Phys. B480 (1996) 265, hep-th/9606139.

[36] M. Dine and N. Seiberg, Nucl. Phys. B301 (1988) 357;

T. Banks and L.J. Dixon, Nucl. Phys. B307 (1988) 93.

[37] I. Antoniadis, C. Bachas, D. Lewellen and T. Tomaras, Phys. Lett. B207 (1988)441.

[38] J. Polchinski, Phys. Rev. Lett. 75 (1995) 4724.

[39] For reviews see J. Polchinski, TASI96, hep-th/9611050;

W. Taylor, in proceedings of the summer school on Particle Physics and Cosmology (Trieste, 1997), hep-th/9801182 ;

C. Bachas, in Gauge Theories, Applied Supersymmetry and Quantum Gravity L.A. Sevrin et al eds. (Imperial College Press, 1996), hep-th/9701019 ; and in last ref. [1], hep-th/9806199.

[40] E. Witten, Nucl.Phys. B460 (1996) 541, hep-th/9511030.

[41] P. Horava and E. Witten, Nycl.Phys. B460 (1996) 506, hep-th/9510209; and Nucl.Phys. B475 (1996) 94, hep-th/9603142.

[42] V. Rubakov and M. Shaposhnikov, Phys. Lett. B125 (1983) 136 ;

G.W. Gibbons and D.L. Wiltshire, Nucl. Phys. B287 (1987) 717.

[43] G. Pradisi and A. Sagnotti, Phys. Lett. B216 (1989) 59;

J. Dai, R.G. Leigh and J. Polchinski, Mod. Phys. Lett. A4 (1989) 2073;

P. Horava, Phys. Lett. B231 (1989) 251 ;

M. Bianchi and A. Sagnotti, Phys.Lett. B247 (1990) 517.

[44] V.A. Kuzmin, I. Tkachev, M. Shaposhnikov, JETP Lett. 36 (1982) 59 ;

J.E. Moody and F. Wilczek, Phys. Rev. D30 (1984) 130;

A. De Rujula, Phys. Lett. B180 (1986) 213.

[45] T.R. Taylor and G. Veneziano, Phys. Lett. B213 (1988) 450 ;

J. Ellis, N.C. Tsamis and M. Voloshin, Phys.Lett. B194 (1987) 291.

S. Dimopoulos and G. F. Giudice, Phys.Lett. B379 (1996) 105, hep-ph/9602350 and references therein.

[46] A. Kehagias and K. Sfetsos, hep-ph/9905417

[47] N. Arkani-Hamed and S. Dimopoulos, hep-ph/9811353.

[48] G.F. Giudice, R. Rattazzi and J.D. Wells, Nucl.Phys. B544 (1999) 3, hep-ph/9811291;

E.A. Mirabelli, M. Perelstein and M.E. Peskin, Phys.Rev.Lett. 82 (1999) 2236, hep$\mathrm{ph} / 9811337$;

T. Han, J.D. Lykken, R.-J. Zhang, Phys.Rev. D59 (1999) 105006, hep-ph/9811350;

J.L. Hewett, Phys.Rev.Lett. 82 (1999) 4765, hep-ph/9811356:

S. Nussinov and R. Shrock, Phys.Rev. D59 (1999) 105002, hep-ph/9811323.

[49] See for example H.P. Nilles, Phys. Rep. 110C (1984) 1.

[50] A. Hashimoto and I. Klebanov, Nucl.Phys.Proc.Suppl. B55 (1997) 118, hep-th/9611214.

[51] L. E. Ibáñez, hep-ph/9905349.

[52] N. Arkani-Hamed, S. Dimopoulos and J. March-Russell, hep-th/9908146.

[53] G. Aldazabal, A. Font, L. E. Ibanez and G. Violero, Nucl. Phys. B536 (1998) 29 ;

L. E. Ibanez, R. Rabadan and A. M. Uranga, Nucl. Phys. B542 (1999) 112 :

I. Antoniadis, C. Bachas and E. Dudas, Nucl. Phys.B560 (1999) 93, hep-th/9906039, 